\title{
Al-based Analysis of Oral Lesions Using Novel Deep Convolutional Neural Networks for Early Detection of Oral Cancer
}

\author{
Kritsasith Warin ( $\square$ warin@tu.ac.th ) \\ Thammasat University \\ Wasit Limprasert \\ Thammasat University \\ Siriwan Suebnukarn \\ Thammasat University \\ Suthin Jinaporntham \\ Khon Kaen University \\ Patcharapon Jantana \\ StoreMesh \\ Sothana Vicharueang \\ StoreMesh
}

\section{Research Article}

Keywords: oral cancer, precancerous conditions, artificial intelligence, deep learning, neural networks

Posted Date: November 9th, 2021

DOI: https://doi.org/10.21203/rs.3.rs-1049349/v1

License: () (1) This work is licensed under a Creative Commons Attribution 4.0 International License. Read Full License 


\section{Abstract}

Background: Artificial intelligence (Al) applications in oncology have been developed rapidly with reported successes in recent years. This work aims to evaluate the performance of deep convolutional neural network (CNN) algorithms for the classification and detection of oral potentially malignant disorders (OPMDs) and oral squamous cell carcinoma (OSCC) in oral photographic images.

Methods: A dataset comprising 980 oral photographic images was divided into 365 images of OSCC, 315 images of OPMDs and 300 images of non-pathological images. Multiclass image classification models were created by using DenseNet-169, ResNet-101, SqueezeNet and Swin-S. And multiclass object detection models were fabricated by using faster R-CNN, YOLOv5, RetinaNet and CenterNet2.

Results: The AUC of multiclass image classification of CNN models was 0.71-1.00 and 0.80- 0.98 on OSCC and OPMDs, respectively. The AUC of multiclass CNN-base object detection models was 0.81-0.91 and 0.34-0.64 on OSCC and OPMDs, respectively. In comparison, CNN- based classification models exhibited a sensitivity and specificity of $0.72-0.99,0.83-0.99$ on OSCC and $0.74-0.95$, 0.88-0.97 on OPMDs, respectively. These values were inline with the performance of oral and maxillofacial surgeons and superior to those of general practictioners (GPs).

Conclusions: CNN-based models have potential for the identification of OSCC and OPMDs in oral photographic images and are expected to be a diagnostic tool to assist GPs for the early detection of oral cancer.

\section{Background}

The power and potential of artificial intelligence (AI) innovations in healthcare are increasingly proven by the desire to improve the quality of clinical care. Novel Al technologies can help clinicians reduce human errors and increase the accurate decision-making with superior outcomes compared to traditional methods [1]. Al applications in head and neck cancer diagnosis have been developed rapidly with reported successes in the initial interpretation of medical images [2]. Among the technological advancements in Al, deep convolutional neural networks (CNN) are the algorithms based on neural networks that mimic the mechanism of human neurons. CNNs are currently being developed as tools to assist clinicians in solving various problems and to increase the accuracy of disease detection in radiographic images and clinical images [3]. The CNN-based algorithms, such as faster R-CNN, ResNet, and DenseNet, have been used to detect and classify lesions in chest x-rays [4] and lesions from clinical images of the skin, cervix, esophagus and larynx, with expert level results [5-8]. The advent of Al technology does not mean the ultimate replacement of clinicians. Instead, it will help clinicians, especially general practitioners (GPs), evaluate and diagnose patients more accurately.

According to the global cancer situation, cancer of the oral cavity, like other life-threatening diseases, is a highly relevant global public health problem. Although oral cancers are the 18th most common cancer worldwide, they are a fatal disease which caused over 170,000 deaths in the year 2020 [9]. Oral squamous cell carcinoma (OSCC) is one frequent malignancy in the oral cavity which accounts for about $90 \%$ of all oral cancers [10]. Two-thirds of oral cancers have been found in developing and low to middle income countries, especially in Southeast Asia and South Asia[9]. Most cases of OSCC are transformed from precancerous lesions of the oral cavity such as erythroplakia, leukoplakia, erythroleukoplakia, oral lichen planus, etc., which are referred to as oral potentially malignant disorders (OPMDs). OPMDs have approximately a 1\% potential to transform into a malignancy lesion [11]. OPMDs and early stages of OSCC are often asymptomatic and may appear as harmless lesions so they may be easily misrecognized, especially by general practictioners (GPs) [12], which leads to delayed diagnosis. Treatment of oral cancer depends on the cancer staging. The advance stages of oral cancer often involves more invasive treatment which increases morbidity, cost of treatment and significantly impacts the individual's quality of life [13]. The prognosis of oral cancer worsens in the advanced stages of cancer. The 5-year survival rate of early stage oral cancer is approximately $69.3 \%$ but will decrease to $31.2 \%$ in the advanced stage [14]. This number has not significantly improved in the past few decades regardless of various treatments [10].

Early detection of OPMD, or early stage of oral cancer, is therefore very important as it not only increases the survival rate but also improves the quality of life of patients. The aim of this study is to evaluate the performance of CNN-based algorithms for the classification and detection of OPMD and OSCC in oral photographic images. The automatic classification performance of the algorithms was compared to that performed by experts (board-certified oral and maxillofacial surgeons) and GPs. These automatic models, combined with clinical data, are expected to provide a new diagnostic tool for GPs to improve the accuracy of early detection of cancerous lesions and to support expert-level decision making in the oral cancer screening program. 


\section{Methods}

\section{Data description}

All clinical oral photographs analyzed in this study were retrospectively collected from an oral and maxillofacial surgery center for a period from January 2009 to December 2020. The oral photographic images were captured from various oral cavity areas. The images were of varying resolutions, the largest was $4496 \times 3000$ pixels and the smallest was 1081 × 836 pixels. The dataset of 980 images was divided into 365 images of OSCC, 315 images of OPMDs and 300 images of non-pathological oral images. The non-pathological oral images were defined as an image of oral mucosa which showed no pathological lesions, e.g., pigmented lesions, OPMDs and malignant lesions.

The reference data used in this study were clinical oral photographs of OSCC, OPMDs and non-pathological oral images which were located in various areas of the oral cavity including buccal mucosa, tongue, upper /lower alveolar ridge, floor of mouth, retromolar trigone and lip. All of the OSCC and OPMDs images were biopsy proven as the gold standard for diagnosis. The OSCC images, which are OSCC stage I-IV according to the TNM clinical staging system as proposed by the American Joint Committee on Cancer (AJCC) [15], and OPMDs images used for analysis in this study were oral leukoplakia, erythroplakia, and erythroleukoplakia with the pathological results of mild, moderate and severe epithelial dysplasia and hyperkeratosis. The image of white striae and erythematous lesion surrounded with white striae had the pathological result of oral lichen planus.

\section{Experiment}

All photographic images were uploaded to the VisionMarker server and web application for image annotation (Digital Storemesh, Bangkok, Thailand). The public version is available on GitHub (GitHub, Inc., CA, USA). The lesion boundaries of the OSCC and OPMDs images were annotated by three oral and maxillofacial surgeons. Owing to the differences in manual labeling from one surgeon to another, the ground truth used was the largest area of intersection between all of the surgeons' annotations in the CNN training, validation and testing (Fig. 1).

\section{Image classification}

Image classification refers to computer algorithms that can classify an image into a certain class according to its visual content. In this work, the CNN-based image classification networks, DenseNet-169, ResNet-101, SqueezeNet and Swin-S, were adopted to create the multiclass image classification model of "OSCC" and "OPMDs" apart from non-pathological oral images on oral photographic images. The image classification experiment was tested on Google Colab (Google Inc., CA, USA) using a Tesla P100, Nvidia driver: 460.32 and CUDA: 11.2 (Nvidia Corporation, CA, USA). The images were preprocessed by augmentation using Keras ImageDataGenerator (opensource software) then the framework resized input images to $224 \times 224$ pixels to feed into a neural network. The neural network architectures in this experiment are DenseNet-169, ResNet-101, SqueezeNet and Swin-S. DenseNet-169 and ResNet-101 are pre-trained weight from ImageNet except SqueezeNet and Swin-S which are pre-trained from scratch. The DenseNet-169, ResNet-101, SqueezeNet and Swin-S were modified to have 2-dimension output vectors, for multiclass: OSCC, OPMDs and non-pathological oral image, with softmax activation function. The hyper parameters used in this study were as follows: maximum number of epochs was 43 , batch size of 32 and learning rate was 0.00001 , except for Swin-S which had maximum number of epochs of 100 and batch size of 16 . The validation loss was very close to the training loss, and there was no significant indication of over-fitting. The details of each image classification algorithm were as follows:

- Densely Connected Convolutional Networks (DenseNet) was proposed by Huang et al. [16] as a CNN-based classification algorithm which connects all layers (with matching feature-map sizes) directly with each other. DenseNet exploits the potential of the network through feature reuse, yielding condensed models that are easy to train and highly parameter efficient which is a good feature extractor for various computer vision tasks that build on convolutional features.

- Residual Networks (ResNet) was developed by He et al. [17] as an architecture that is implemented by reformulating the layers as learning residual functions with reference to the layer inputs. This residual learning framework can gain more accuracy of object classification from considerably increased depth, producing results substantially better than previous networks.

- SqueezeNet was proposed by landola et al. [18] as a small CNN architecture with model compression techniques to less than 0.5 MB by decreasing the quantity of parameters and maximizing accuracy on a limited budget of parameters. SqueezeNet had $50 x$ fewer parameters than a previous CNN, AlexNet, but maintained AlexNet-level accuracy. 
- Swin Transformer (Swin) was presented by Liu et al. [19] as a new vision transformer which produces a hierarchical feature representation and has linear computational complexity with respect to input image size. The design of Swin as a shifted window based self-attention is shown to be effective and efficient on image classification.

\section{Object detection}

Detecting of lesions is another key to success in disease diagnosis. The CNN-based object detection is shown to be effective in identifying disease in the image. In this study, Faster R-CNN, YOLOv5, RetinaNet and CenterNet2 were adopted to detect the OSCC and OPMDs lesions in oral photographic images. The object detection experiment used the annotated image from VisionMarker (Digital Storemesh, Bangkok, Thailand). The annotated images were identified by bounding boxes showing locations of the lesion areas; then the pairs of image and annotation were ready for the training process. The image was preprocessed by augmentation using Keras ImageDataGenerator (open-source software) then the framework resized an input image to $256 \times 256$ pixels, except YOLOv 5 which resized an input image to $640 \times 640$ pixels, to feed into a neural network. The training was performed on an on-premise server with 2 of GPU, TitanXP 12GB, Nvidia driver: 450.102 and CUDA: 11.0 (Nvidia Corporation, CA, USA). The neural network architectures were FasterR-CNN, YOLOv5, RetinaNet and CenterNet2 with the pre-trained weight from COCO Detection. All the networks were trained using stochastic gradient descent (SGD). The hyper parameters used in this study were as follows: 20,000 iterations, maximum number of epochs was 1,882 , learning rate of 0.0025 and batch size per image of 128 , except for YOLOv 5 which had maximum number of epochs of 200 , learning rate of 0.01 and batch size per image of 8 . The training loss was reduced and maintained between 15,000 and 20,000 iterations. The details of each object detection algorithm were as follows:

- Faster regional convolutional neural network (Faster R-CNN) was introduced by Ren et al. [20] as a CNN-based object detection framework. This algorithm is the combination of the previous object detection system, Fast R-CNN, and Region Proposal Networks (RPNs) into a single network to share their convolutional features leading to a more real-time object detection method. This design significantly improved the speed and accuracy in the object detection compared to basis R-CNN.

- You only look once (YOLO) was proposed by Redmon et al. [21] as a CNN-based object detection algorithm which reframes as a single regression problem, straight from image pixels to bounding box coordinates and class probabilities. The YOLO design enables end-to-end training and realtime speeds while maintaining high average precision.

- RetinaNet was proposed by Lin et al. [22] as a simple one-stage object detector with a new loss function that acts as a more effective alternative to previous algorithms for dealing with class imbalance. This design achieves state of-the-art accuracy and speed for the object detection.

- CenterNet2 was developed by Zhou et al. [23] as a probabilistic interpretation of two-stage detectors. This algorithm was designed as a simple modification of standard two-stage detector training by optimizing a lower bound to a joint probabilistic objective over both stages which achieved desirable speed and accuracy for the object detection.

To evaluate the performance of the image classification and object detection networks, five-fold cross-validation was employed. Data elements were split into 5 subsets using random sampling with equal numbers of OSCC, OPMDs and non-pathological oral images. Then, one subset was considered as a testing set, while the remaining four subsets were used as training and validation sets. This process was repeated 5 times to involve all subsets as testing sets.

\section{Evaluation measures}

The metrics used to evaluate the machine learning algorithms in bioinformatics were used in this study [24]. The CNN-based image classification models were evaluated using the precision, recall (sensitivity), specificity, F1 score, and area under the receiver operating characteristics curve (AUC of ROC) to measure the performance in classifying OSCC and OPMDs on the oral photographic images. The classification performance of models was also evaluated by generating a heat map visualization using the gradient-weighted class activation mapping (Grad-CAM) [25] to see how the models classify and identify OSCC and OPMDs on photographic images. For the object detection, the performance of the CNN-based object detection models was evaluated to detect a bounding box relative to the ground truth region in the OSCC and OPMDs images by the precision, recall, F1 score and AUC of precision-recall curve. If the loU value between the generated bounding box and the ground truth was less than 0.5 , then the produced bounding box was considered to be a false prediction or false positive.

A test dataset with known pathological results was evaluated to compare the performance of the CNN-based classification models with that of 20 clinicians; 10 board certified oral and maxillofacial surgeons and 10 GPs who have at least 2 years of experience in dental 
practice in rural hospitals. None of these readers participated in the clinical care or assessment of the enrolled patients, nor did they have access to their medical records. The overall sensitivity and specificity of these clinicians were calculated. Data analyses were conducted using SPSS version 22.0 (SPSS, Chicago, IL). The statistical analysis for image classification and object detection was calculated as follows:

$\mathrm{IOU}=$ area of overlap/area of union

Precision $=$ TP $/ T P+F P$

Recall (Sensitivity) $=$ TP/TP + FN

Specificity $=\mathrm{TN} / \mathrm{TN}+\mathrm{FP}$

F1 score $=2 \times($ Precision $\times$ Recall $) /$ Precision + Recall

- True positive (TP): positive outcomes that the model predicted correctly which loU $>0.5$.

- False positive (FP): positive outcomes that the model predicted incorrectly which loU $<0.5$.

- True negative (TN): negative outcomes that the model predicted correctly.

- False negative (FN): negative outcomes that the model predicted incorrectly.

\section{Results}

Image classification results

The evaluation of multiclass images was performed on the test set and the results of the CNN-based image classification models are reported in Table 1. The image classification of CNN-based image classification models achieved a precision, a recall (sensitivity), a specificity, an F1 score and AUC of ROC curve as seen in Table 2. The overall sensitivity and specificity for the classification by the ten oral and maxillofacial surgeons of OCSS were $0.90(95 \% \mathrm{Cl}=0.85-0.96)$ and $0.89(95 \% \mathrm{Cl}=0.81-0.97)$ and OPMDs were $0.74(95 \% \mathrm{Cl}$ $=0.61-0.87)$ and $0.93(95 \% \mathrm{Cl}=0.90-0.96)$, respectively. In addition, the overall sensitivity and specificity for the classification by the ten GPs of OCSS were $0.77(95 \% \mathrm{Cl}=0.70-0.85)$ and $0.87(95 \% \mathrm{Cl}=0.85-0.90)$ and OPMDs were $0.68(95 \% \mathrm{Cl}=0.62-0.75)$ and $0.86(95 \% \mathrm{Cl}$ $=0.82-0.90$ ), respectively. Fig. 2 shows an example of the Grad-CAM visualization of the DenseNet-169 output of OSCC and OPMDs classes which shows that the model correctly classifies and identifies OSCC and OPMDs on photographic images.

Table 1

Multi-class image classification performances of CNN algorithms on the test sets compared with the average performance of clinicians; 'Oral and maxillofacial surgeons' vs. 'GPs'

\begin{tabular}{|c|c|c|c|c|c|c|c|c|c|c|}
\hline & \multicolumn{10}{|l|}{ Class } \\
\hline & \multicolumn{5}{|l|}{ OScC } & \multicolumn{5}{|l|}{ OPMDs } \\
\hline & Precision & $\begin{array}{l}\text { Recall } \\
\text { (Sensitivity) }\end{array}$ & Specificity & $\begin{array}{l}\text { F1 } \\
\text { score }\end{array}$ & $\begin{array}{l}\text { AUC } \\
\text { of } \\
\text { ROC } \\
\text { curve }\end{array}$ & Precision & $\begin{array}{l}\text { Recall } \\
\text { (Sensitivity) }\end{array}$ & Specificity & $\begin{array}{l}\text { F1 } \\
\text { score }\end{array}$ & $\begin{array}{l}\text { AUC } \\
\text { of } \\
\text { ROC } \\
\text { curve }\end{array}$ \\
\hline $\begin{array}{l}\text { DenseNet- } \\
169\end{array}$ & 0.98 & 0.99 & 0.99 & 0.98 & 1.0 & 0.95 & 0.95 & 0.97 & 0.95 & 0.98 \\
\hline ResNet-101 & 0.96 & 0.92 & 0.94 & 0.94 & 0.99 & 0.97 & 0.97 & 0.94 & 0.97 & 0.97 \\
\hline SqueezeNet & 0.85 & 0.72 & 0.92 & 0.78 & 0.88 & 0.76 & 0.78 & 0.88 & 0.77 & 0.87 \\
\hline Swin-S & 0.69 & 0.73 & 0.83 & 0.71 & 0.71 & 0.63 & 0.74 & 0.88 & 0.68 & 0.80 \\
\hline $\begin{array}{l}\text { Oral and } \\
\text { maxillofacial } \\
\text { surgeons }\end{array}$ & - & 0.90 & 0.89 & - & - & - & 0.74 & 0.93 & - & - \\
\hline GPs & - & 0.77 & 0.87 & - & - & - & 0.68 & 0.86 & - & - \\
\hline
\end{tabular}


Table 2

Multi-class object detection performances of CNN algorithms on the test sets

\begin{tabular}{|c|c|c|c|c|c|c|c|c|}
\hline & \multicolumn{8}{|l|}{ Class } \\
\hline & \multicolumn{4}{|l|}{ oscc } & \multicolumn{4}{|l|}{ OPMDs } \\
\hline & Precision & Recall & $\begin{array}{l}\text { F1 } \\
\text { score }\end{array}$ & $\begin{array}{l}\text { AUC of precision - recall } \\
\text { curve }\end{array}$ & Precision & Recall & $\begin{array}{l}\text { F1 } \\
\text { score }\end{array}$ & $\begin{array}{l}\text { AUC of precision - recall } \\
\text { curve }\end{array}$ \\
\hline $\begin{array}{l}\text { Faster R- } \\
\text { CNN }\end{array}$ & 0.84 & 0.90 & 0.87 & 0.88 & 0.60 & 0.71 & 0.65 & 0.64 \\
\hline YOLOv5 & 0.88 & 0.86 & 0.87 & 0.84 & 0.74 & 0.39 & 0.51 & 0.34 \\
\hline RetinaNet & 0.98 & 0.82 & 0.89 & 0.81 & 0.92 & 0.57 & 0.70 & 0.55 \\
\hline CenterNet2 & 0.64 & 0.92 & 0.76 & 0.91 & 0.49 & 0.60 & 0.54 & 0.58 \\
\hline
\end{tabular}

\section{Object detection results}

The object detection models were evaluated on the test set and the results are reported in Table 2. The detection performance of CNNbased object detection models achieved a precision, a recall, an F1 score and AUC of precision-recall curve as shown in Table 2. Examples of detection outputs from CNN-based object detection models in this study are provided in Fig. 3.

\section{Discussion}

Oral cancer screening is an important part of an oral examination, the goal of which is to identify changes and the development of oral cancer. It is commonly known that OSCC, the most common oral cancer, is often preceded by OPMDs [11]. Delay in diagnosing oral cancer may lead to more invasive treatment resulting in greater morbidity of oral functions, such as distortions of speech, chewing and swallowing, which will have a significant impact on individual's quality of life [13]. Usually, when diagnosed at an advanced stage, less than $50 \%$ of oral cancer patients survive more than 5 years. This rate has remained disappointingly low and relatively constant during the last few decades $[10,14]$. Therefore, the early detection of oral cancer, especially OPMDs or early stage OSCC, with appropriate referral to specialists is crucial to control the disease and improve the survival rate and quality of life of patients. Screening of oral cancer is largely based on visual examination. Advances in computer vision and Al technology that improve visual detection can be used to assist visual examination combined with clinical data as a novel diagnostic tool in the oral cancer screening system.

In this work, the performance of CNN-based image classification models works well to identify the OSCC and OPMDs. The results, particularly in DenseNet-169 and ResNet-101, achieved near-perfect AUC and showed performance similar to the classification of multiclass image of OSCC and OPMDs on oral images as a CNN model of the study of Fu et al. [26] but better than the studies of Welikala et al. [27] The difference in the performance of models may be from variations in the class distribution of each study. DenseNet-169 and ResNet-101 are a series of well-optimized algorithms, which achieve high performance in image classification, and are widely used in the medical field. However, the DenseNet-169 and ResNet-101 algorithms were a large CNN architecture and required a high-performance computing server for the image classification processing which may not be appropriate for use in a mobile application for oral cancer screening. Therefore, this work selected new and smaller CNN models, SqueezeNet and Swin-S, to test the classification performance of OSCC and OPMDs on oral photographic images. SqueezeNet and Swin-S showed acceptable accuracy and achieved an AUC of 0.71-0.88 which may have inferior performance than DenseNet-169 and ResNet-101. But the small size architecture of these models was more suited for developing into a mobile application for oral cancer screening. In the medical field, there was a study that successfully used SqueezeNet for the diagnosis of the coronavirus disease 2019 (COVID-19) from chest X-ray images [28]. To the best of our knowledge, this is the first study to use Swin-S for classification of oral lesions. These CNN-based classification models yield a classification performance of OSCC and OPMDs on oral photograph equal to expert level (board certified oral and maxillofacial surgeons) and superior to GP level.

For the detection of oral lesions, the CNN-based object detection used in this study showed good performance in the detection of OSCC and OPMDs on photographic images which achieved AUC of 0.81-0.91 and 0.34-0.64 in the detection of OSCC and OPMDs, respectively. One of the generally CNN-based object detection algorithms used in medical images, the faster R-CNN achieved high

Page 6/12 
performance in the detection of OSCC and OPMDs with AUC of 0.88 and 0.64 , respectively. The faster R-CNN detection performance in this study achieved higher precision, recall and F1 score than the previous study of Welikala et al. [27] for detecting the OSCC and OPMDs on oral photographs which may be from the different number of classes in the study. Nowadays, there is a continuous development of $\mathrm{CNN}$-based object detection to increase the accuracy of detection of the interested object. CenterNet2, one of the latest CNN-based object detection models, achieved the highest performance in detection of OSCC, an AUC of 0.91, but was slightly inferior to faster R-CNN for detecting of OPMDs. The overall OPMDs performance in detection in this study was not as good as the detection of OSCC which may result from the general characteristics of OPMDs in the oral cavity which make them difficult to recognize, even by the expert. The lowest performance model in detection of OPMDs is YOLOv5 which achieved an AUC of 0.34 . This may be due to YOLOv5 being an extremely fast detection model with an operating time of only 0.07 seconds per frame [21]. A high-speed model of this type may not be appropriate for detecting the features of OPMDs on oral images.

As the focus of $\mathrm{Al}$ is shifting from model/algorithm development to the quality of the data used to train the models [29], this study has limitations that need to be addressed. First, the dataset was small and only included OSCC and OPMDs images. And second, the process of labeling lesions on oral photographic images required experts to identify the ground truth on the images, which was time consuming. For future work, we plan to develop the CNN-based mobile application to collect more data and expand the image dataset to include other oral lesions such as pigmented lesions and submucosal lesions, from the multi-cancer center and hospitals in a remote area. In addition, we plan to develop the system integrated into the clinical workflow to allow the experts to label the ground truth of the lesion in the image. This not only saves time on the labeling process, but also increases the chances of the experts to thoroughly study the details of the lesion in the image.

\section{Conclusions}

CNN-based models showed comparable diagnostic performances to expert level in classifying OSCC and OPMDs on oral photographic images. This is expected to be a novel innovation as a diagnostic tool to assist clinicians, especially GPs, in improving the accuracy of early detection of cancerous lesions and support expert-level decision making in the oral cancer screening program.

\section{Abbreviations}

Al: Artificial intelligence; CNN: Deep convolutional neural network; OPMDs: Oral potentially malignant disorders; OSCC: oral squamous cell carcinoma; GPs: General practictioners; DenseNet: Densely Connected Convolutional Networks; ResNet: Residual Networks; Swin: Swin Transformer; R-CNN; Regional convolutional neural network; RPNs: Region proposal networks; YOLO: You only look once; AUC: Area under the curve; ROC: Receiver operating characteristics.

\section{Declarations}

\section{Acknowledgements}

We gratefully acknowledge the support of the NVIDIA Corporation for the Titan Xp GPU (Nvidia Corporation, CA, USA) used in this research. We thank Waranthorn Chansawang for their assistance with the CNN model training.

\section{Author contributions}

KW: conceptualization, methodology, validation, formal analysis, investigation, data curation, resources, writing - original draft, writing review \& editing, visualization, project administration, funding acquisition. WS: methodology, software, validation, formal analysis. SS: conceptualization, methodology, validation, writing - review \& editing, visualization, supervision. SJ: resources. PJ and SV: software, validation.

\section{Funding}

This work was supported by the Thammasat University Research Grant (TUFT 24/2564) and the Health Systems Research Institute.

\section{Availability of data and materials}

The datasets used and/or analyzed during the current study are available from the corresponding author on reasonable requests. 


\section{Ethics approval and consent to participate}

The study has been approved by the institute research ethics committee (COE 020/2563) in accordance with the 1964 Helsinki declaration and its later amendments.

\section{Consent for publication}

Not applicable.

\section{Competing interests}

The authors declare no competing interests.

\section{References}

1. McKinney SM, Sieniek M, Godbole V, Godwin J, Antropova N, Ashrafian H, Back T, Chesus M, Corrado GS, Darzi A et al: International evaluation of an Al system for breast cancer screening. Nature 2020, 577(7788):89-94.

2. Mahmood H, Shaban M, Rajpoot N, Khurram SA: Artificial Intelligence-based methods in head and neck cancer diagnosis: an overview. Br J Cancer 2021, 124(12):1934-1940.

3. Litjens G, Kooi T, Bejnordi BE, Setio AAA, Ciompi F, Ghafoorian M, van der Laak J, van Ginneken B, Sanchez Cl: A survey on deep learning in medical image analysis. Med Image Anal 2017, 42:60-88.

4. Faes L, Wagner SK, Fu DJ, Liu X, Korot E, Ledsam JR, Back T, Chopra R, Pontikos N, Kern C et al: Automated deep learning design for medical image classification by health-care professionals with no coding experience: a feasibility study. Lancet Digit Health 2019, 1(5):e232-e242.

5. Ghatwary N, Zolgharni M, Ye X: Early esophageal adenocarcinoma detection using deep learning methods. Int J Comput Assist Radiol Surg 2019, 14(4):611-621.

6. Xiong H, Lin P, Yu JG, Ye J, Xiao L, Tao Y, Jiang Z, Lin W, Liu M, Xu J et al: Computer-aided diagnosis of laryngeal cancer via deep learning based on laryngoscopic images. EBioMedicine 2019, 48:92-99.

7. He X, Wang S, Shi S, Tang Z, Wang Y, Zhao Z, Dai J, Ni R, Zhang X, Liu X et al: Computer-Aided Clinical Skin Disease Diagnosis Using CNN and Object Detection Models. In: 2019 IEEE International Conference on Big Data (Big Data): 9-12 Dec. 2019 2019; 2019: 4839-4844.

8. Park YR, Kim YJ, Ju W, Nam K, Kim S, Kim KG: Comparison of machine and deep learning for the classification of cervical cancer based on cervicography images. Sci Rep 2021, 11(1):16143.

9. Sung H, Ferlay J, Siegel RL, Laversanne M, Soerjomataram I, Jemal A, Bray F: Global cancer statistics 2020: GLOBOCAN estimates of incidence and mortality worldwide for 36 cancers in 185 countries. CA Cancer J Clin 2021.

10. Warnakulasuriya S: Global epidemiology of oral and oropharyngeal cancer. Oral Oncol 2009, 45(4-5):309-316.

11. Warnakulasuriya S: Oral potentially malignant disorders: A comprehensive review on clinical aspects and management. Oral Oncol $2020,102: 104550$.

12. McCormick NJ, Thomson PJ, Carrozzo M: The Clinical Presentation of Oral Potentially Malignant Disorders. Prim Dent J 2016, 5(1):52-63.

13. Shah JP, Gil Z: Current concepts in management of oral cancer-surgery. Oral Oncol 2009, 45(4-5):394-401.

14. Chen SW, Zhang Q, Guo ZM, Chen WK, Liu WW, Chen YF, Li QL, Liu XK, Li H, Ou-Yang D et al: Trends in clinical features and survival of oral cavity cancer: fifty years of experience with 3,362 consecutive cases from a single institution. Cancer Manag Res 2018, 10:4523-4535.

15. Amin MB, Edge S, Greene FL, Schilsky RL, Byrd DR, Gaspar LE, Washington MK, Gershenwald JE, Compton CC, Hess KR: AJCC Cancer Staging Manual, $8^{\text {th }}$ edn: Springer Nature; 2017.

16. Huang G, Liu Z, Maaten LVD, Weinberger KQ: Densely Connected Convolutional Networks. In: 2017 IEEE Conference on Computer Vision and Pattern Recognition (CVPR): 21-26 July 2017 2017; 2017: 2261-2269.

17. He K, Zhang X, Ren S, Sun J: Deep Residual Learning for Image Recognition. In: 2016 IEEE Conference on Computer Vision and Pattern Recognition (CVPR): 27-30 June 2016 2016; 2016: 770-778. 
18. landola FN, Han S, Moskewicz MW, Ashraf K, Dally WJ, Keutzer K: SqueezeNet: AlexNet-level accuracy with 50x fewer parameters and $<0.5 \mathrm{MB}$ model size. In.; 2016.

19. Liu Z, Lin Y, Cao Y, Hu H, Wei Y, Zhang Z, Lin S, Guo B: Swin Transformer: Hierarchical Vision Transformer using Shifted Windows. ArXiv 2021, abs/2103.14030.

20. Ren S, He K, Girshick R, Sun J: Faster R-CNN: Towards Real-Time Object Detection with Region Proposal Networks. IEEE Transactions on Pattern Analysis and Machine Intelligence 2017, 39(6):1137-1149.

21. Redmon J, Divvala S, Girshick R, Farhadi A: You Only Look Once: Unified, Real-Time Object Detection. In: 2016 IEEE Conference on Computer Vision and Pattern Recognition (CVPR): 27-30 June 2016 2016; 2016: 779-788.

22. Lin T, Goyal P, Girshick R, He K, Dollár P: Focal Loss for Dense Object Detection. IEEE Transactions on Pattern Analysis and Machine Intelligence 2020, 42(2):318-327.

23. Zhou X, Koltun V, Krähenbühl P: Probabilistic two-stage detection. ArXiv 2021, abs/2103.07461.

24. Jiao Y, Du P: Performance measures in evaluating machine learning based bioinformatics predictors for classifications. Quantitative Biology 2016, 4(4):320-330.

25. Selvaraju RR, Cogswell M, Das A, Vedantam R, Parikh D, Batra D: Grad-CAM: Visual Explanations from Deep Networks via GradientBased Localization. In: 2017 IEEE International Conference on Computer Vision (ICCV): 22-29 0ct. 2017 2017; 2017: 618-626.

26. Fu Q, Chen Y, Li Z, Jing Q, Hu C, Liu H, Bao J, Hong Y, Shi T, Li K et al: A deep learning algorithm for detection of oral cavity squamous cell carcinoma from photographic images: A retrospective study. EClinicalMedicine 2020, $27: 100558$.

27. Welikala RA, Remagnino P, Lim JH, Chan CS, Rajendran S, Kallarakkal TG, Zain RB, Jayasinghe RD, Rimal J, Kerr AR et al: Automated Detection and Classification of Oral Lesions Using Deep Learning for Early Detection of Oral Cancer. IEEE Access 2020, 8:132677-132693.

28. Ucar F, Korkmaz D: COVIDiagnosis-Net: Deep Bayes-SqueezeNet based diagnosis of the coronavirus disease 2019 (COVID-19) from X-ray images. Med Hypotheses 2020, 140:109761.

29. Min E, Guo X, Liu Q, Zhang G, Cui J, Long J: A Survey of Clustering With Deep Learning: From the Perspective of Network Architecture. IEEE Access 2018, 6:39501-39514.

\section{Figures}




\section{Annotation}

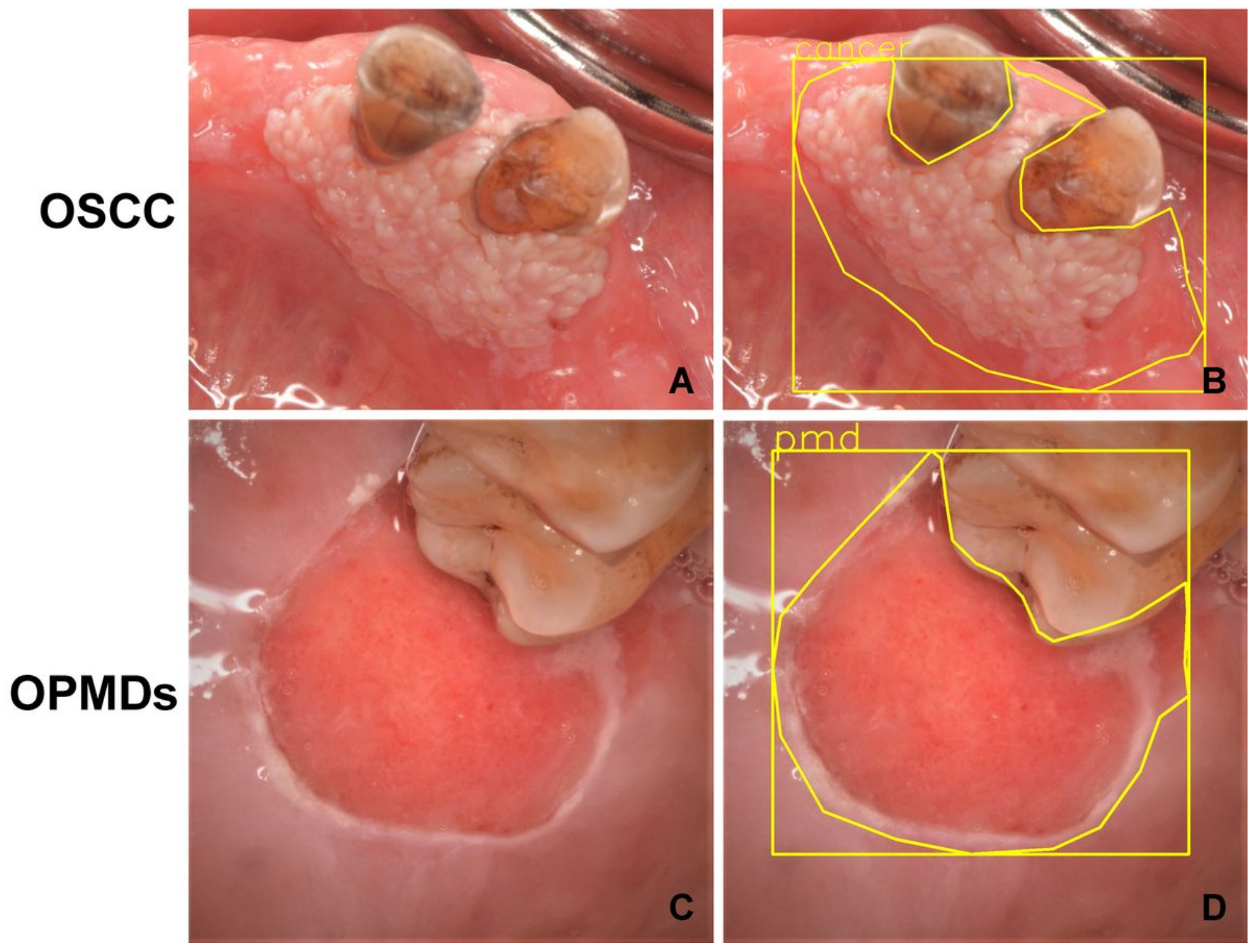

\section{Figure 1}

Examples of the OSCC and OPMDs images from the dataset showing. A OSCC image; B annotation of OSCC image by surgeons; C OPMDs image; $\mathrm{D}$ annotation of OPMDs image by surgeons 


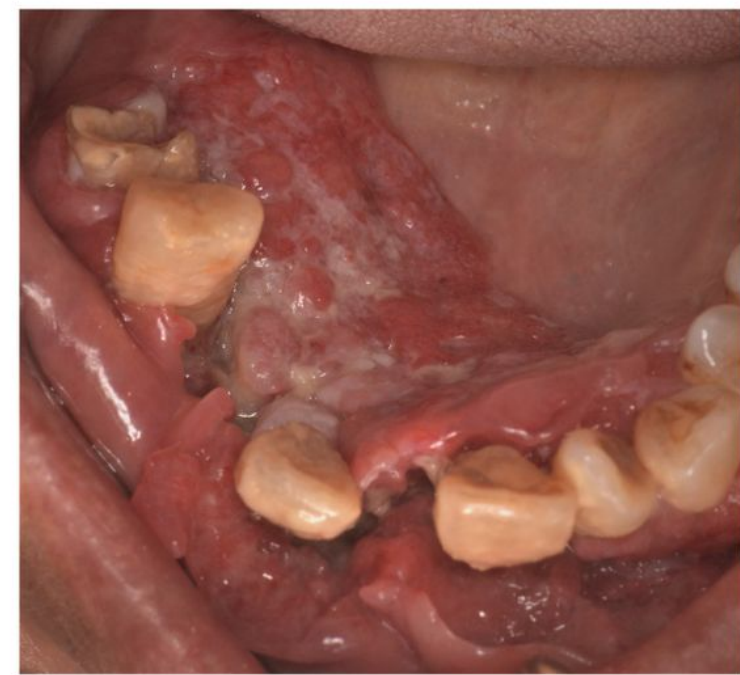

(A) Input OSCC image

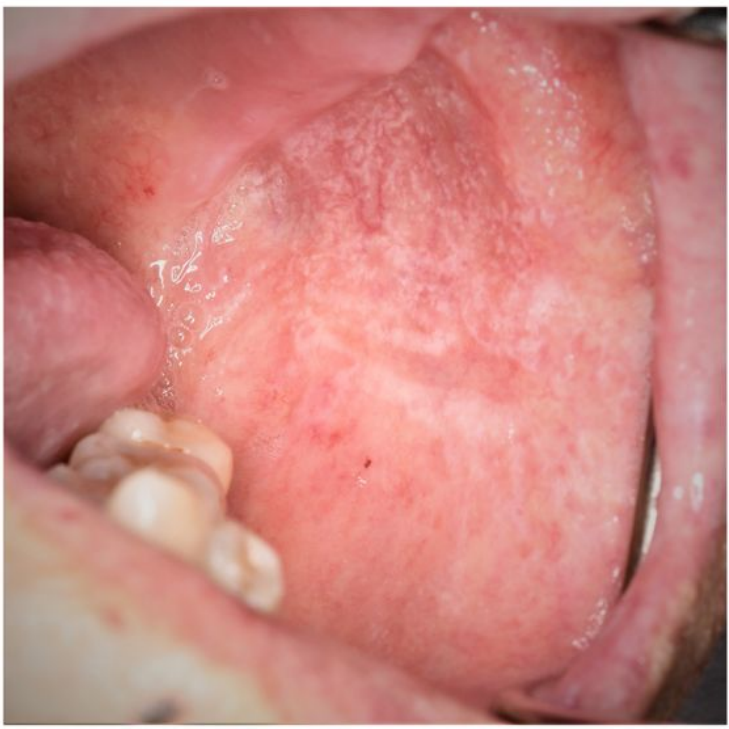

(C) Input OPMDs image

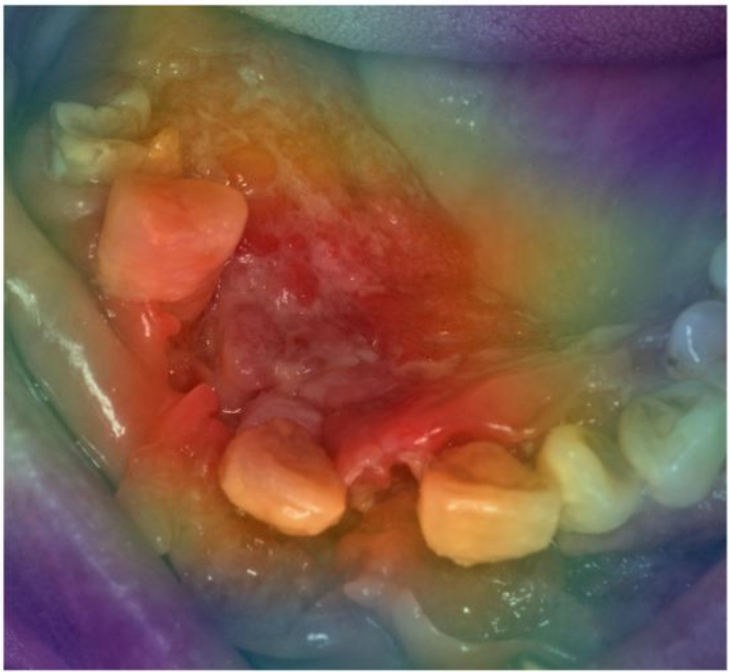

(B) Grad-CAM

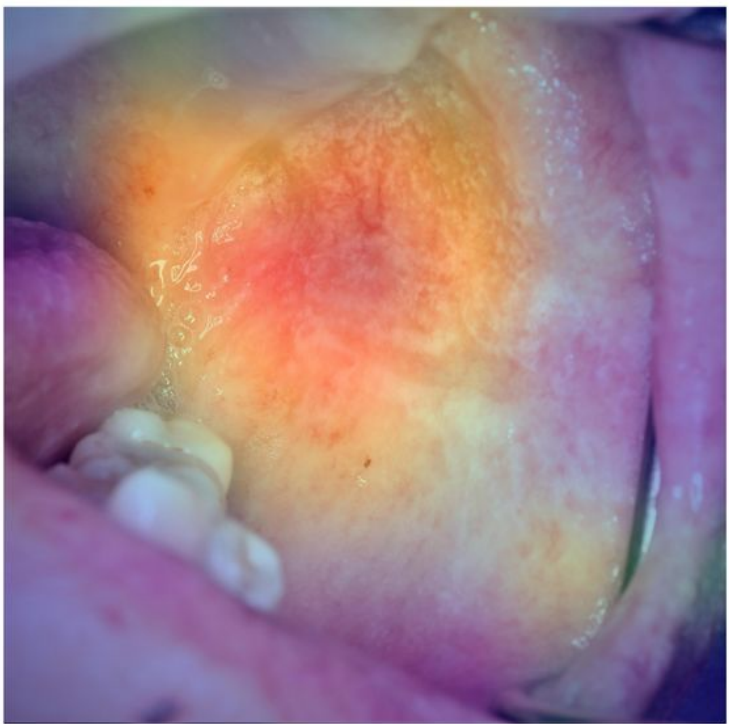

(D) Grad-CAM

\section{Figure 2}

Example of the Grad-CAM visualization of the DenseNet-169. A Image with OSCC lesion; B The model correctly classified OSCC and labeled the correct location. C Image with OPMDs lesion D The model correctly classified OPMDs and labeled the correct location 


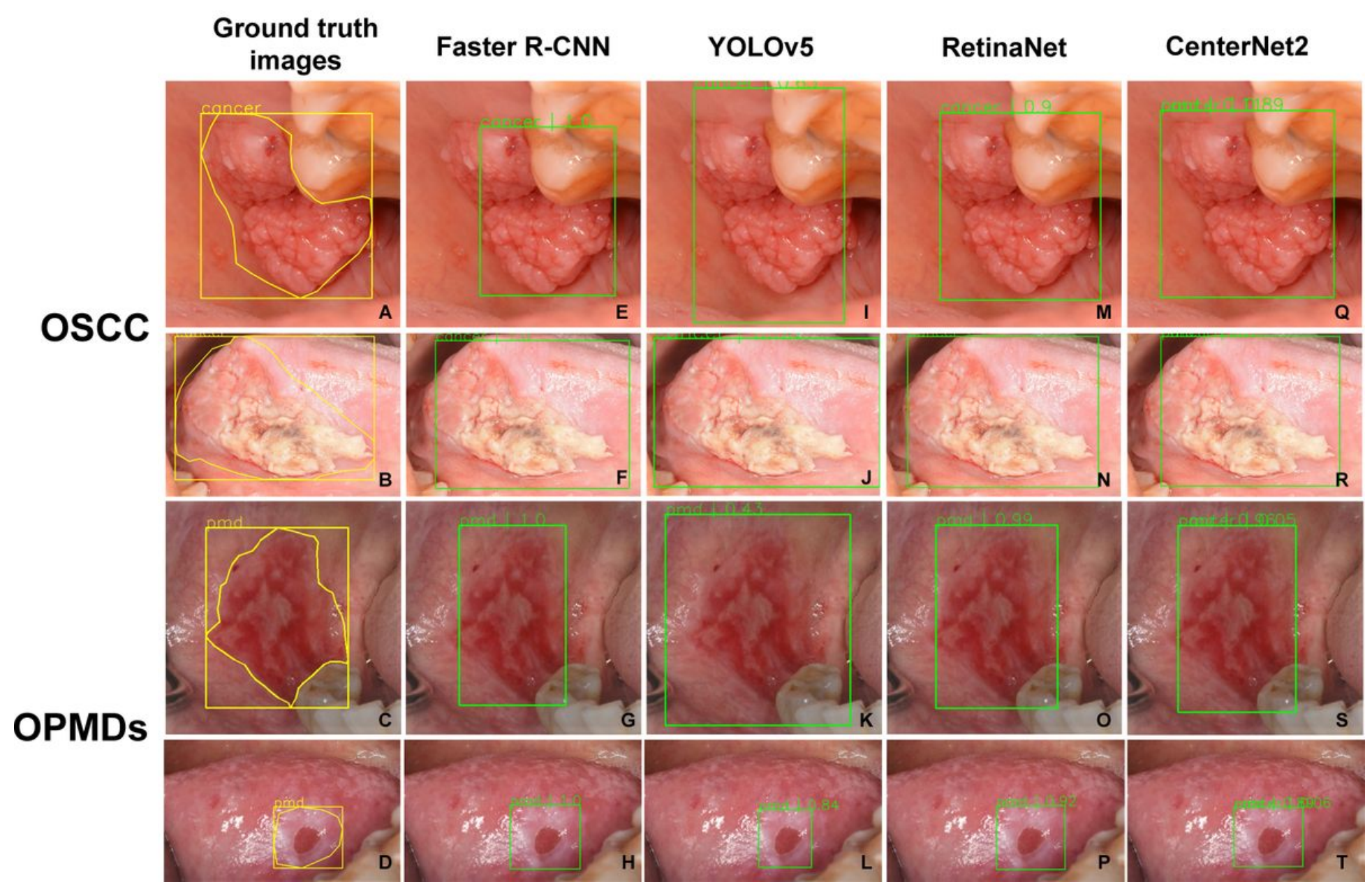

Figure 3

A-B Bounding box ground truth based on surgeons' annotations of the imaging of the patient with OSCC at retromolar trigone and lateral tongue, respectively; C-D Bounding box ground truth based on surgeons' annotations of the imaging of the patient with OPMDs at retromolar trigone and lateral tongue, respectively; E-H The true positive outputs from the faster R-CNN detection; I-L The true positive outputs from the YOLOv5 detection; M-P The true positive outputs from the RetinaNet detection; Q-T The true positive outputs from the CenterNet2 detection 